Bundesgesundheitsbl 2012 $\cdot 55: 102-110$

DOI 10.1007/s00103-011-1401-3

Online publiziert: 24. Dezember 2011

(c) Springer-Verlag 2011
T. Lampert · G.B.M. Mensink · S. Müters

FG27 Gesundheitsberichterstattung, Robert Koch-Institut, Berlin
Ergebnisse der Studie "Gesundheit in Deutschland aktuell 2009"
Regelmäßige körperliche Aktivität leistet einen wichtigen Beitrag zum Erhalt beziehungsweise zur Wiedererlangung der Gesundheit. Eine Vielzahl wissenschaftlicher Studien zeigt, dass die körperliche Aktivität sowohl bei der Vermeidung vorzeitiger Sterblichkeit als auch bei der Vermeidung, Behandlung und Rehabilitation von Herz-Kreislauf-Erkrankungen, Schlaganfall, Adipositas, Bluthochdruck, Diabetes mellitus Typ 2, Osteoporose, Rückenschmerzen und verschiedenen Krebserkrankungen eine wesentliche Rolle spielt $[1,2,3,4]$. Außerdem finden sich Hinweise darauf, dass regelmäßige körperliche Aktivität zur Vorbeugung beziehungsweise Linderung von Depressionen beiträgt und den Erhalt der mentalen Fähigkeiten bis ins hohe Lebensalter unterstützt $[1,5]$. Auch der Sport ist mit gesundheitsförderlichen Potenzialen verbunden, die sich nicht nur über physiologische Prozesse, sondern auch im $\mathrm{Zu}$ sammenhang mit der Stressregulation, der Ausformung personaler und sozialer Kompetenzen sowie der Entwicklung einer insgesamt gesünderen Lebensweise entfalten können $[6,7]$. Während körperliche Aktivität jede körperliche Bewegung umfasst, die durch die Skelettmuskulatur produziert wird und den Energieverbrauch über den Grundumsatz anhebt, bezeichnet Sport eine spezifische Form der körperlichen Aktivität, für die insbesondere körperliche Leistung, Wettkampf und Spaß an der Bewegung typisch sind [6]. Die vorliegenden Erkenntnisse sprechen dafür, dass durch bewegungs- und sportfördernde Maßnahmen und Programme, insbesondere wenn diese auf die Mobilisierung der Bevölkerungsgruppen zielen, die sich nur wenig körperlich und sportlich betätigen, die Morbidität und vorzeitige Sterblichkeit in der Bevölkerung erheblich vermindert werden können [2].

Eine wichtige Grundlage für die Planung, Umsetzung und Evaluation politischer und praxisbezogener Maßnahmen sind regelmäßig bereitgestellte Daten zum Aktivitätsverhalten und zur Sportbeteiligung der Bevölkerung. Am Robert Koch-Institut (RKI) wurde in den letzten Jahren ein Gesundheitsmonitoring etabliert, das eine kontinuierliche Beobachtung des Gesundheitszustandes und des Gesundheitsverhaltens in Deutschland sicherstellen soll. Das Gesundheitsmonitoring umfasst drei Komponenten: die Studie „Gesundheit in Deutschland aktuell“ (GEDA), eine regelmäßige telefonische Befragung der erwachsenen Bevölkerung in Deutschland, die Studie „Gesundheit von Erwachsenen in Deutschland“ (DEGS), die als Wiederholungsbefragung zum Bundes-Gesundheitssurvey 1998 konzipiert ist und neben Querschnitt- auch Längsschnittdaten bereitstellen wird, sowie die Fortführung des Kinder- und Jugendgesundheitssurveys
(KiGGS) als Kohortenstudie [8]. Das Erhebungsprogramm der Studien ist breit angelegt und umfasst auch die körperlich-sportliche Aktivität. Im Folgenden werden die Daten der GEDA-Studie 2009 genutzt, um die körperlich-sportliche Aktivität der erwachsenen Bevölkerung in Deutschland zu untersuchen. Dabei werden alters-, geschlechts- und sozialstatusspezifische Unterschiede ausgewiesen, die mögliche Ansatzpunkte für zielgruppenorientierte Maßnahmen der Bewegungs- und Sportförderung aufzeigen. Außerdem wird auf zeitliche Entwicklungen und Trends eingegangen und dazu auf Daten der früheren Gesundheitssurveys des RKI zurückgegriffen. Möglich ist dies allerdings nur für die sportliche Aktivität, da die körperliche Aktivität in den vorausgegangenen Surveys nicht in vergleichbarer Weise erhoben wurde.

\section{Daten und Methode}

Bei der Studie „Gesundheit in Deutschland aktuell“ (GEDA) handelt es sich um eine bundesweit repräsentative telefonische Befragung, die das Robert Koch-Institut (RKI) im Auftrag des Bundesministeriums für Gesundheit (BMG) durchführt $[8,9]$. Die Grundgesamtheit der GEDA-Studie ist die 18-jährige und ältere Wohnbevölkerung Deutschlands, die in Privathaushalten lebt und über einen Festnetzanschluss verfügt. Als Stichpro- 
benbasis dient ein nach dem Gabler-Häder-Design [10] erstelltes Telefonnummernsample, das auch die Grundlage für das Mastersample des Arbeitskreises Deutscher Marktforschungsinstitute (ADM) liefert und für fast alle bundesweit repräsentativen Telefonbefragungen in Deutschland herangezogen wird [11, 12]: Dabei werden in dem um Service-, Fax- und gewerbliche Nummern bereinigten Nummernpool die letzten beiden Ziffern abgeschnitten und durch zufällige Ziffernfolgen von „oo“ bis „99“ ersetzt. Das Gabler-Häder-Design entspricht damit im Kern einem Random-Route-Ansatz und gewährleistet auch die Einbeziehung von Haushalten, die nicht in öffentlich zugänglichen Telefonverzeichnissen eingetragen sind. Die Repräsentativität auf Zielpersonenebene wird sichergestellt, indem in Mehrpersonenhaushalten diejenige volljährige Person befragt wird, die zuletzt Geburtstag hatte (Last-Birthday-Methode).

Der Erhebungszeitraum der GEDAStudie 2009 erstreckte sich vom 15 . Juli 2008 bis zum 5. Juni 2009. In diesem Zeitraum wurden insgesamt 21.262 Telefoninterviews realisiert. Das Kooperations- und Antwortverhalten wird in der GEDA-Studie 2009 gemäß der international standardisierten Vorgaben der American Association for Public Opinion Research [13] untersucht und bewertet. Die Kooperationsrate auf Zielpersonenebene (Anteil der realisierten Interviews an allen Zielpersonenkontakten) lag in der GEDA-Studie 2009 bei 51,2\% [9, 14].

Die Telefoninterviews wurden computerunterstützt von geschulten Interviewern und Interviewerinnen in einem eigens am RKI dafür eingerichteten Telefonlabor durchgeführt. Die verwendete Software VOXCO Interviewsuite 5.4.4.5 erlaubt eine komfortable und zeitökonomische Interviewführung. $\mathrm{Zu}$ den Themenschwerpunkten der GEDA-Studie 2009 zählten chronische Erkrankungen und Beschwerden, die subjektive Gesundheit und gesundheitsbezogene Lebensqualität, das Gesundheitsverhalten und assoziierte Risikofaktoren, Ressourcen der Gesundheit, wie zum Beispiel soziale Beziehungen und Nutzung von Gesundheitsinformationen, die Inanspruchnahme des Gesundheitswesens, insbe-

Bundesgesundheitsbl 2011 · 55:102-110 DOI 10.1007/s00103-011-1401-3

() Springer-Verlag 2011

\section{T. Lampert · G.B.M. Mensink · S. Müters}

Körperlich-sportliche Aktivität bei Erwachsenen in Deutschland. Ergebnisse der Studie „Gesundheit in Deutschland aktuell 2009"

\section{Zusammenfassung}

Analysiert wird die Verbreitung der körperlichen und sportlichen Aktivität in der erwachsenen Bevölkerung Deutschlands. Die Datenbasis wird durch die Studie "Gesundheit in Deutschland aktuell 2009" gebildet, für die vom Robert Koch-Institut im Zeitraum von Juli 2008 bis Juni 2009 insgesamt 21.262 Telefoninterviews durchgeführt wurden. Demnach sind $56 \%$ der 18 -jährigen und älteren Männer und $38 \%$ der gleichaltrigen Frauen mindestens 2,5 Stunden pro Woche so aktiv, dass sie dabei ins Schwitzen oder außer Atem geraten. Dass sie in den letzten drei Monaten Sport getrieben haben, trifft den Selbstangaben zufolge auf jeweils 64\% der Männer und Frauen zu. Im Vergleich zu den Jahren 1998 und 2003 zeigt sich, dass die Sportbeteiligung bei Männern und Frauen in allen Alters- gruppen zugenommen hat. Darüber hinaus sind statusspezifische Unterschiede zu beobachten, bei der körperlichen Aktivität zuungunsten der hohen Statusgruppen, bei der sportlichen Aktivität zuungunsten der niedrigen Statusgruppen. Ansatzpunkte für bewegungs- und sportfördernde Maßnahmen ergeben sich insbesondere in Bezug auf Personen, die an keinem Tag in der Woche körperlich aktiv sind (19\% der Männer und 26\% der Frauen) und keinen Sport treiben (jeweils $36 \%$ der Männer und Frauen).

\section{Schlüsselwörter}

Körperliche Aktivität · Sport .

Gesundheitsverhalten - Gesundheitssurvey . Gesundheitsmonitoring

\section{Physical and sporting activity among adults in Germany. Results from the "German Health Update 2009" survey}

\section{Abstract}

The study analyses the distribution of physical and sporting activity among the adult population in Germany. The data came from the "German Health Update 2009" survey, which is based on 21,262 telephone interviews conducted by the Robert Koch Institute between July 2008 and June 2009. The study shows that $56 \%$ of men aged 18 and over and $38 \%$ of women in the same age group engaged in physical activity with an intensity that made them sweat or be out of breath for at least $2.5 \mathrm{~h} /$ week. In addition, $64 \%$ of both men and women stated that they had actively engaged in sporting activities over the previous 3 months. Comparing 1998 and 2003, participation in sport was observed to have increased among men and women in all age groups over time. Furthermore, socio-economic differences were observed, indicating lower physical activity among higher-status groups and lower sporting activity among lower-status groups. Measures to promote physical activity and sport prove to be especially necessary for people who are not physically active (19\% of men and $26 \%$ of women) or who do not engage in sport at all (36\% of both men and women).

\section{Keywords}

Physical activity · Sports · Health-related behaviour $\cdot$ Health surveys $\cdot$ Health monitoring 
80

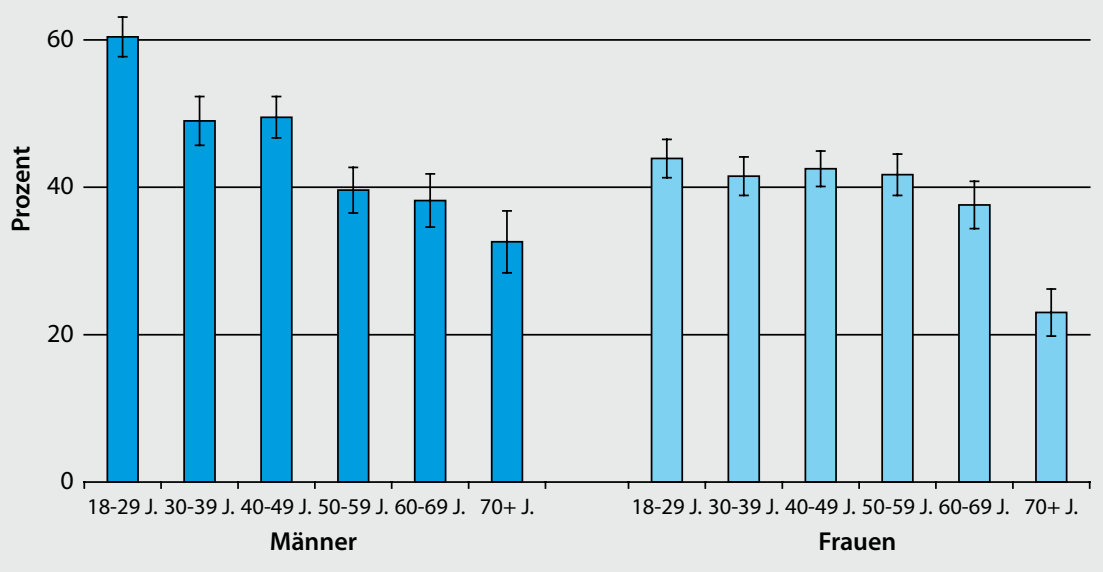

Abb. $1 \Delta$ Anteil der Männer und Frauen, die mindestens 2,5 Stunden pro Woche körperlich aktiv sind nach Alter (Prävalenzen mit 95\%-Konfidenzintervallen, $\mathrm{n}=20.761$ )

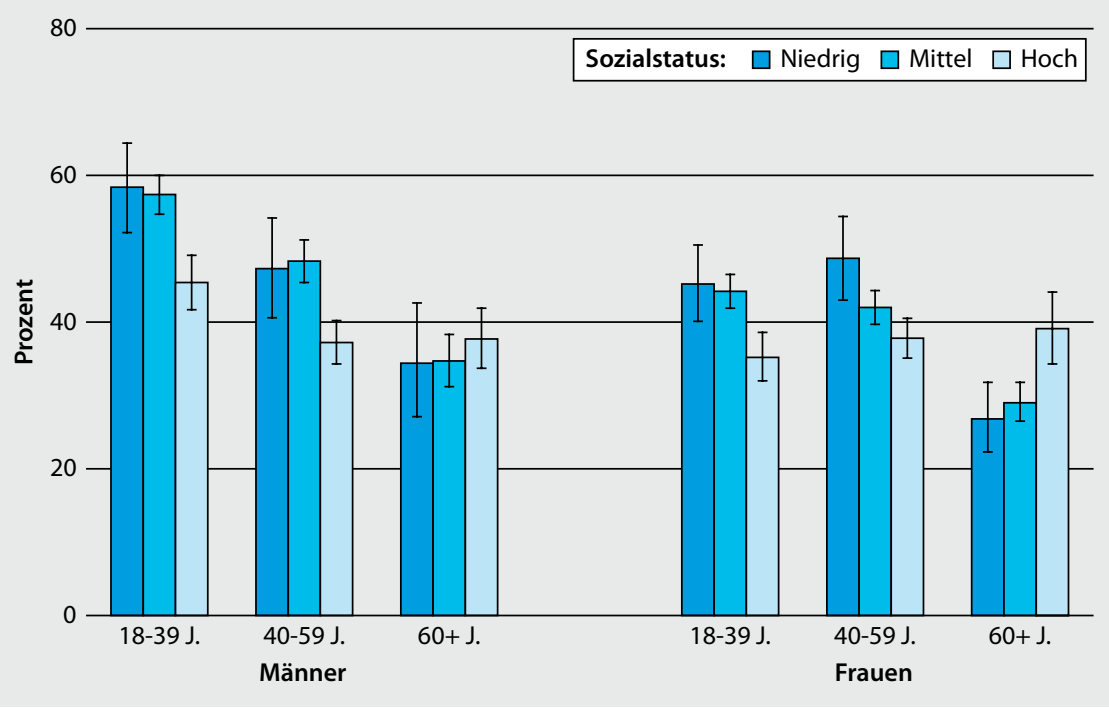

Abb. $2 \Delta$ Anteil der Männer und Frauen, die mindestens 2,5 Stunden pro Woche körperlich aktiv sind, nach Sozialstatus und Alter (Prävalenzen mit 95\%-Konfidenzintervallen, $n=20.673$ )

sondere in Bezug auf Präventionsangebote, sowie die Soziodemografie und Lebensbedingungen [9].

Die körperliche und sportliche Aktivität wurde in der GEDA-Studie 2009 mit separaten Frageblöcken erfasst. Bezüglich der körperlichen Aktivität sollten die Befragten zunächst angeben, an wie vielen Tagen in der Woche sie körperlich so aktiv sind, dass sie ins Schwitzen oder außer Atem kommen. Anschließend sollten sie einschätzen, wie lange sie sich an diesen Tagen durchschnittlich körperlich betätigen (Antwortkategorien: ,weniger als zehn Minuten“, „zehn bis unter 30 Minuten“, „30 bis unter 6o Minuten“, „60 Mi- nuten und mehr"). Auf Basis dieser Informationen wurde für die Analysen ein Indikator mit vier Ausprägungen gebildet: „kaum körperliche Aktivität“, „bis 2,5 Stunden pro Woche aktiv", „2,5 oder mehr Stunden pro Woche aktiv, an weniger als fünf Tagen aktiv“, „mindestens an fünf Tagen pro Woche aktiv, jeweils 30 oder mehr Minuten“. Der Gruppe mit „kaum körperlicher Aktivität“ wurden alle Personen zugeordnet, die an keinem Tag in der Woche körperlich so aktiv sind, dass sie dabei ins Schwitzen oder außer Atem kommen. Die Unterscheidung zwischen den beiden zuletzt genannten Kategorien beruht auf den zum
Zeitpunkt der Planung der GEDA-Studie 2009 geltenden Empfehlungen der US-amerikanischen Centers of Disease Control and Prevention (CDC). Danach sollten sich Erwachsene an mindestens fünf Tagen in der Woche für mindestens 3o Minuten körperlich so betätigen, dass sie dabei ins Schwitzen geraten und die Atemfrequenz steigt $[1,15]$.

Auch die sportliche Aktivität wurde zweistufig erhoben. Zunächst sollten die Studienteilnehmer angeben, ob sie in den letzten drei Monaten Sport gemacht haben (Antwortkategorien: „ja“, "nein“). Die Personen, die diese Frage bejahten, wurden außerdem gefragt, wie viele Stunden in der Woche sie sich sportlich betätigen (Antwortkategorien: „bis zu einer Stunde“, „bis zu zwei Stunden“, „bis zu drei Stunden“, „bis zu vier Stunden“, „mehr als vier Stunden“). Der für die Analysen auf Basis der Antworten auf beide Fragen gebildete Indikator hat vier Ausprägungen: ,keine sportliche Aktivität", „bis zu zwei Stunden pro Woche aktiv“, „Zwei bis vier Stunden pro Woche aktiv", „vier und mehr Stunden pro Woche aktiv". Zu berücksichtigen ist dabei, dass die Frageformulierungen keine Rückschlüsse darauf erlauben, wie regelmäßig die Befragten das angegebene Wochenpensum absolvieren. Deshalb kann eine Überschätzung der sportlichen Aktivität nicht ausgeschlossen werden, da zumindest ein Teil der Befragten nicht jede Woche die angegebene Stundenzahl erreichen dürfte.

Die Analysen wurden für Männer und Frauen getrennt durchgeführt und dabei zwischen sechs Altersgruppen (18 bis 29,30 bis 39,40 bis 49,50 bis 59,60 bis $69,70+$ Jahre) unterschieden. Außerdem werden Unterschiede in der körperlichen und sportlichen Aktivität nach dem Sozialstatus berichtet. Der Sozialstatus wurde in der GEDA-Studie 2009 auf Basis von Informationen zur schulischen und beruflichen Bildung, zur beruflichen Stellung und zum Netto-Äquivalenzeinkommen (nach der neuen OECD-Äquivalenzskala bedarfsgewichtetes Haushaltsnettoeinkommen) in zwei Schritten ermittelt. Zunächst wurde ein Punktsummenscore berechnet, in den die Einzelindikatoren gleichwertig eingingen. Im Anschluss wurde eine verteilungsba- 
sierte Abgrenzung von drei Statusgruppen („niedrig“, „mittel“, „hoch“) vorgenommen, wobei die niedrige und hohe Statusgruppe jeweils $20 \%$ und die mittlere Statusgruppe 60\% der Befragten umfassten [16].

Die statistischen Analysen wurden mit dem Statistikprogramm PASW (Predictive Analytics Software) Statistics 18 von SPSS durchgeführt. Neben Prävalenzen mit 95\%-Konfidenzintervallen werden im Folgenden zum Teil auch altersadjustierte Odds Ratios ausgewiesen, die mittels binär logistischer Regressionen berechnet wurden. Die Odds Ratios beziehen sich zum einen auf den Einfluss des sozialen Status auf die körperliche beziehungsweise sportliche Aktivität und bringen damit zum Ausdruck, um welchen Faktor die Chance für körperliche beziehungsweise sportliche Aktivität in der mittleren und hohen im Verhältnis zur niedrigen Statusgruppe (Referenzkategorie) erhöht ist. Zum anderen wurde eine binär logistische Regression für die Veränderung der Chance für sportliche Aktivität im Zeitraum von 1998 bis 2009 durchgeführt. Die Odds Ratios besagen in diesem Fall, um welchen Faktor sich die Chance für sportliche Aktivität im Jahr 2009 im Verhältnis zum Jahr 1998 (Referenzkategorie) verändert hat. $\mathrm{Ob}$ diese Veränderung statistisch signifikant war, wurde anhand der ebenfalls ausgewiesenen p-Werte beurteilt. Den p-Werten liegt ein Wald-Test zugrunde. Ein p-Wert von 0,05 besagt beispielsweise, dass die Nullhypothese, der zufolge sich die betrachtete Gruppe in Bezug auf die Outcomevariable nicht signifikant von der definierten Referenzgruppe unterscheidet, bei einer zuvor spezifizierten Irrtumswahrscheinlichkeit von $\alpha=0,05$ verworfen werden kann.

Wie ein Abgleich mit der amtlichen Bevölkerungsstatistik zeigt, sind Männer, ältere Menschen (ab 7o Jahre) und Personen mit niedrigen Bildungsabschlüssen in der Stichprobe der GEDA-Studie 2009 unterrepräsentiert. Außerdem konnten geringe Abweichungen auf der Ebene der Bundesländer festgestellt werden. Um die design- und responsebedingten Verzerrungen in der Stichprobe auszugleichen und die Repräsentativität der Ergebnisse zu verbessern, wurden alle Analysen mit Gewichtungsfaktoren durchgeführt,

Tab. 1 Ausmaß der körperlichen Aktivität von Männern und Frauen nach Alter (Prävalenzen in \% mit 95\%-Konfidenzintervallen) $(n=20.761)$

\begin{tabular}{ll}
\multicolumn{2}{l}{ Körperliche Aktivität } \\
Kaum körper- & Weniger als \\
liche Aktivität ${ }^{\mathrm{a}}$ & 2,5 Stunden pro \\
& Woche aktiv
\end{tabular}

Männer

\begin{tabular}{|lllll}
\hline 18-29 Jahre & $7,5(6,2-9,1)$ & $32,1(29,5-34,7)$ & $32,0(29,4-34,7)$ & $28,4(25,9-31,1)$ \\
\hline 30-39 Jahre & $14,1(11,8-16,7)$ & $36,9(33,9-40,0)$ & $21,7(19,1-24,5)$ & $27,3(24,4-30,4)$ \\
\hline 40-49 Jahre & $12,8(11,1-14,9)$ & $37,7(35,0-40,4)$ & $22,6(20,3-25,1)$ & $26,9(24,3-29,6)$ \\
\hline 50-59 Jahre & $21,0(18,5-23,8)$ & $39,4(36,4-42,5)$ & $18,5(16,1-21,1)$ & $21,1(18,7-23,7)$ \\
\hline 60-69 Jahre & $27,0(23,8-30,5)$ & $34,8(31,4-38,3)$ & $22,8(19,8-26,0)$ & $15,4(12,9-18,2)$ \\
\hline 70+ Jahre & $40,4(36,2-44,6)$ & $27,0(23,4-31,0)$ & $16,5(13,6-19,8)$ & $16,2(13,2-19,7)$ \\
\hline Gesamt & $18,9(17,8-20,0)$ & $35,0(33,8-36,3)$ & $22,8(21,7-23,9)$ & $23,3(22,2-24,5)$ \\
\hline Frauen & & & & \\
\hline 18-29 Jahre & $13,5(11,8-15,4)$ & $42,6(40,1-45,1)$ & $22,5(20,4-24,8)$ & $21,4(19,3-23,6)$ \\
\hline 30-39 Jahre & $18,4(16,4-20,5)$ & $40,1(37,6-42,7)$ & $17,4(15,5-19,4)$ & $24,1(21,9-26,5)$ \\
\hline 40-49 Jahre & $18,2(16,4-20,1)$ & $39,3(37,1-41,6)$ & $18,7(17,0-20,5)$ & $23,8(21,7-26,1)$ \\
\hline 50-59 Jahre & $23,3(21,0-25,8)$ & $34,9(32,4-37,6)$ & $18,3(16,4-20,5)$ & $23,4(21,1-25,9)$ \\
\hline 60-69 Jahre & $29,4(26,4-32,6)$ & $33,0(30,0-36,2)$ & $21,7(19,0-24,5)$ & $15,9(13,7-18,3)$ \\
\hline 70+ Jahre & $51,3(47,7-54,9)$ & $25,7(22,6-29,0)$ & $11,4(9,4-13,8)$ & $11,6(9,5-14,1)$ \\
\hline Gesamt & $26,0(24,9-27,1)$ & $35,9(34,8-37,0)$ & $18,1(17,2-19,0)$ & $20,0(19,1-21,0)$
\end{tabular}

aKaum körperliche Aktivität meint, dass die betreffenden Personen nach eigenen Angaben an keinem Tag in der Woche körperlich so aktiv sind, dass sie dabei ins Schwitzen oder außer Atem kommen.

Tab. 2 Körperliche Aktivität (2,5 + Stunden pro Woche) und sozialer Status. Ergebnisse binär logistischer Regressionen bei Adjustierung bezüglich des Alters (Odds Ratios mit 95\%-Konfidenzintervallen und $p$-Werten) $(n=20.673)$

\begin{tabular}{|c|c|c|c|c|c|}
\hline & \multicolumn{5}{|c|}{ Sozialstatus } \\
\hline & \multirow[t]{2}{*}{ Niedrig } & \multicolumn{2}{|l|}{ Mittel } & \multicolumn{2}{|l|}{ Hoch } \\
\hline & & OR (95\%-KI) & p-Wert & OR (95\%-KI) & p-Wert \\
\hline \multicolumn{6}{|l|}{ Männer } \\
\hline 18-39 Jahre & Ref. & $0,99(0,75-1,31)$ & $p=0,939$ & $0,67(0,50-0,92)$ & $p<0,010$ \\
\hline 40-59 Jahre & Ref. & $1,02(0,76-1,37)$ & $p=0,806$ & $0,65(0,48-0,87)$ & $p<0,001$ \\
\hline $60+$ Jahre & Ref. & $0,98(0,67-1,45)$ & $p=0,885$ & $1,10(0,75-1,63)$ & $p=0,437$ \\
\hline Gesamt & Ref. & $1,00(0,84-1,20)$ & $p=0,962$ & $0,75(0,62-0,90)$ & $p<0,001$ \\
\hline \multicolumn{6}{|l|}{ Frauen } \\
\hline 18-39 Jahre & Ref. & $0,97(0,76-1,22)$ & $p=0,701$ & $0,67(0,51-0,88)$ & $p<0,010$ \\
\hline 40-59 Jahre & Ref. & $0,76(0,59-0,97)$ & $p<0,010$ & $0,64(0,49-0,82)$ & $p<0,001$ \\
\hline $60+$ Jahre & Ref. & $0,99(0,75-1,32)$ & $p=0,911$ & $1,43(1,03-1,99)$ & $p<0,010$ \\
\hline Gesamt & Ref. & $0,97(0,84-1,13)$ & $p=0,592$ & $0,87(0,75-1,02)$ & $p<0,050$ \\
\hline
\end{tabular}

die anhand eines mehrstufigen Verfahrens ermittelt wurden [9]. In einem ersten Schritt wurden Designgewichte auf Basis der Haushaltsgröße und der Anzahl der Telefonanschlüsse im Haushalt berechnet. Für den designgewichteten Datensatz wurde anschließend eine dreistufige, iterative Randverteilungsanpassung (sogenannte Poststratifizierung) vorgenommen, die sich an der Verteilung der Merkmale Alter, Wohnort (Bundesland) und Bildungsbeteiligung (nach der Internatio- nal Standard Classification of Education, ISCED [17]) in der Grundgesamtheit orientierte. Die Datenbasis bildeten dabei der Mikrozensus 2007 und die laufende Bevölkerungsfortschreibung des Statistischen Bundesamtes (Stand 31.12.2008). Zuerst wurde an die Randverteilung in der Grundgesamtheit nach sieben Altersklassen ( 18 bis 24,25 bis 34,35 bis 44,45 bis 54,55 bis 64,65 bis $69,70+$ ) und Geschlecht angepasst, dann nach sechs Altersklassen ( 18 bis 24,25 bis 39,40 bis 49 , 
100

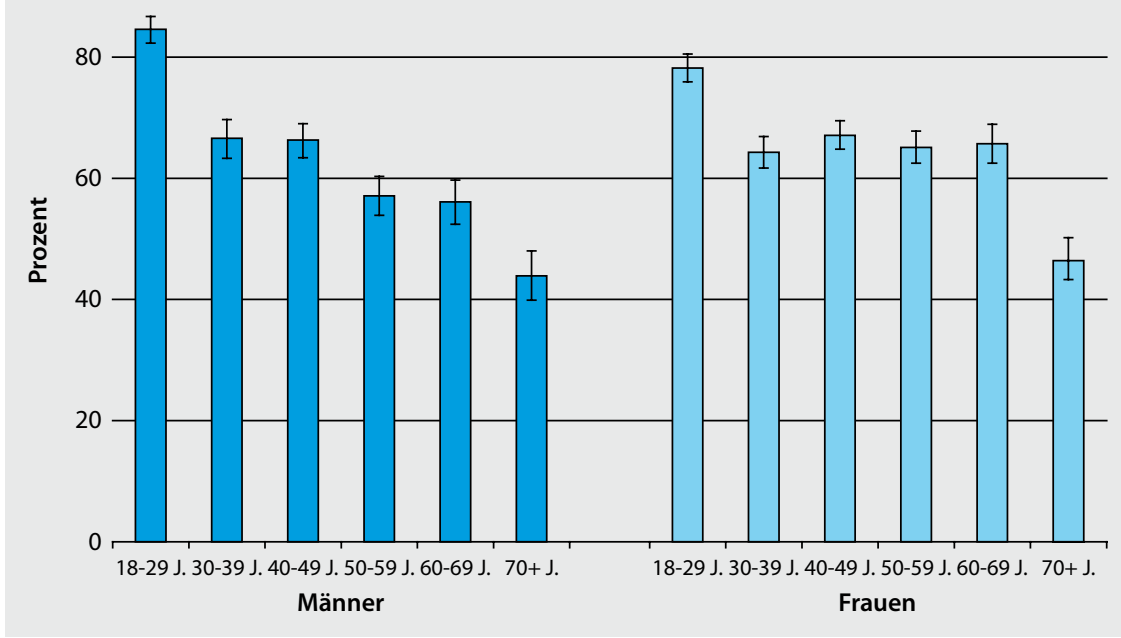

Abb. $3 \Delta$ Anteil der Männer und Frauen, die in den letzten drei Monaten Sport getrieben haben, nach Alter (Prävalenzen mit 95\%-Konfidenzintervallen, $n=21.245$ )

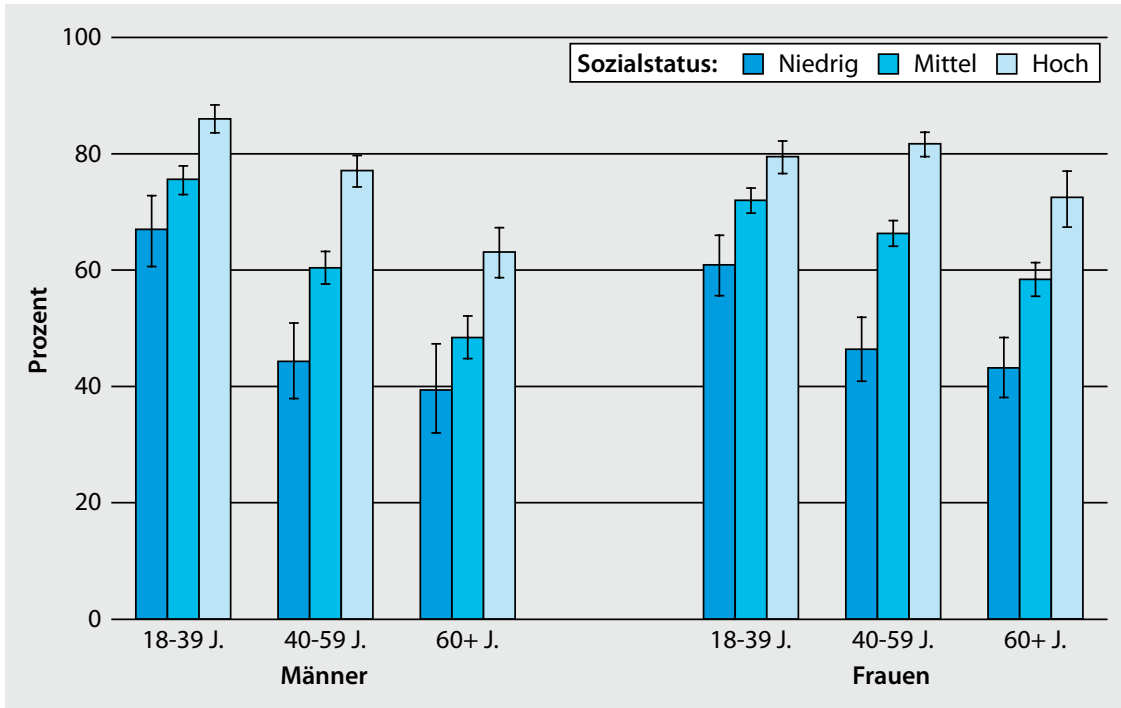

Abb. $4 \Delta$ Anteil der Männer und Frauen, die in den letzten drei Monaten Sport getrieben haben, nach Sozialstatus und Alter (Prävalenzen mit 95\%-Konfidenzintervallen, $n=21.151$ )

50 bis 59,60 bis $69,70+$ ), drei ISCEDKlassen und Geschlecht und zuletzt nach 16 Bundesländern, sechs Altersklassen (18 bis 24,25 bis 39,40 bis 49,50 bis 59,60 bis $69,70+$ ) und Geschlecht. Die auf diese Weise berechneten Gewichtungsfaktoren variieren zwischen 0,27 und 5,40 $(\mathrm{MEAN}=1, \mathrm{Oo} ; \mathrm{SD}=0,86)$.

\section{Ergebnisse}

Bezüglich der körperlichen Aktivität wurde zunächst der Anteil der Personen betrachtet, die mindestens 2,5 Stunden in der Woche aktiv sind. Bei den 18-jährigen und älteren Männern trifft dies auf 56\% und bei den gleichaltrigen Frauen auf 38\% zu. Bei Männern ist der Anteil der körperlich Aktiven im jungen Erwachsenenalter am höchsten und nimmt dann im Altersgang kontinuierlich ab, während sich bei Frauen in Bezug auf das Aktivitätsverhalten im jungen und mittleren Erwachsenenalter nur geringe Unterschiede zeigen und erst bei den 70-Jährigen und Älteren ein deutlicher Rückgang zu beobachten ist (• Abb. 1).

Bei zusätzlicher Berücksichtigung der Anzahl der Tage, an denen die Befragten körperlich aktiv waren, können Aussagen über den Anteil der Personen getroffen werden, die das von der CDC empfohlene Aktivitätsniveau erreichen. Demnach sind 23\% der Männer und 20\% der Frauen im Alter ab 18 Jahren an mindestens fünf Tagen in der Woche jeweils mindestens 30 Minuten körperlich aktiv (• Tab. 1). Außerdem lässt sich feststellen, dass fast jeder fünfte Mann und jede vierte Frau an keinem Tag in der Woche körperlich aktiv ist. Der Anteil der körperlich inaktiven Männer und Frauen steigt mit zunehmendem Alter an und ist folglich in der Gruppe der 70-Jährigen und Älteren am höchsten (40\% beziehungsweise 51\%).

Darüber hinaus sind Unterschiede im Aktivitätsverhalten nach dem sozialen Status zu beobachten, die nachfolgend für drei Altersgruppen ausgewiesen werden. In der Gruppe der 18- bis 39- und der 40bis 59-Jährigen sind mehr Männer und Frauen mit niedrigem Sozialstatus mindestens 2,5 Stunden pro Woche aktiv als Männer und Frauen mit hohem Sozialstatus. Ab einem Alter von 6o Jahren liegt hingegen der Anteil der körperlich Aktiven in der hohen Statusgruppe über dem entsprechenden Anteil in der niedrigen Statusgruppe, was bei Frauen noch deutlicher zum Ausdruck kommt als bei Männern (• Abb. 2).

Bei Adjustierung bezüglich des Alters in einem binär logistischen Regressionsmodell mit der körperlichen Aktivität als abhängiger und dem Sozialstatus als unabhängiger Variable lässt sich die Aussage treffen, dass bei Männern die Chance, mindestens 2,5 Stunden wöchentlich körperlich aktiv zu sein, in der hohen Statusgruppe im Verhältnis zur niedrigen Statusgruppe (Referenzkategorie) um den Faktor 0,75 erhöht, das heißt (umgerechnet) um den Faktor 1,33 verringert ist (- Tab. 2). Auch bei Frauen ist die Chance, körperlich aktiv zu sein, in der hohen gegenüber der niedrigen Statusgruppe verringert. Dies gilt zumindest für das junge und mittlere Erwachsenenalter. In Bezug auf das höhere Lebensalter bestätigt die Regressionsanalyse den deskriptiven Befund einer stärkeren Verbreitung körperlicher Aktivität bei Frauen mit hohem Sozialstatus. Des Weiteren machen die Ergebnisse deutlich, dass weder bei Männern noch bei Frauen signifikante Unterschiede im Aktivitätsverhal- 
ten der Angehörigen der niedrigen und mittleren Statusgruppe bestehen.

Die Ergebnisse der GEDA-Studie 2009 zeigen zudem, dass $64 \%$ der 18 -jährigen und älteren Männer und ein ebenso hoher Anteil der gleichaltrigen Frauen in den letzten drei Monaten Sport getrieben haben. Eine nach Altersgruppen differenzierte Betrachtung macht deutlich, dass bei Männern die sportliche Aktivität, ähnlich wie bei der körperlichen Aktivität, mit zunehmendem Alter sukzessive sinkt. Auch bei Frauen ist die sportliche Aktivität im jungen Erwachsenenalter am höchsten und im fortgeschrittenen Lebensalter am geringsten. Zwischen den mittleren Altersgruppen sind hingegen keine Unterschiede beim Anteil der sportlich Aktiven zu beobachten (• Abb. 3).

Bei Berücksichtigung des für den Sport aufgebrachten Stundenumfangs fällt auf, dass jeweils etwa ein Fünftel der 18-jährigen und älteren Bevölkerung ein bis zwei Stunden, zwei bis vier Stunden beziehungsweise vier und mehr Stunden pro Woche Sport treibt ( $\bullet$ Tab. 3). Dass sie vier oder mehr Stunden pro Woche sportlich aktiv sind, wird von Männern häufiger angegeben als von Frauen. Außerdem wird deutlich, dass etwa ein Drittel der erwachsenen Bevölkerung überhaupt keinen Sport macht. Allerdings bestehen diesbezüglich erhebliche Unterschiede zwischen den Altersgruppen: Im jungen Erwachsenenalter trifft dies auf jeden siebten Mann und jede fünfte Frau zu. Von den 7o-Jährigen und Älteren gibt etwas mehr als die Hälfte an, dass sie keinen Sport ausüben.

Bezüglich der sportlichen Aktivität sind deutliche Unterschiede nach dem sozialen Status festzustellen. Bei Männern wie Frauen ist die Sportbeteiligung in der niedrigen Statusgruppe am geringsten und in der hohen Statusgruppe am höchsten. Dies gilt für alle drei betrachteten Altersgruppen (• Abb. 4).

Bei Adjustierung bezüglich des Alters im binär logistischen Regressionsmodell haben Männer aus der hohen Statusgruppe im Verhältnis zu Männern aus der niedrigen Statusgruppe eine um den Faktor 3,42 erhöhte Chance, sportlich aktiv zu sein. Bei Frauen beträgt das entsprechende Chancenverhältnis 3,53:1. Auch

Tab. 3 Ausmaß der sportlichen Aktivität von Männern und Frauen in den letzten drei Monaten nach Alter (Prävalenzen \% mit 95\%-Konfidenzintervallen) ( $n=21.245)$

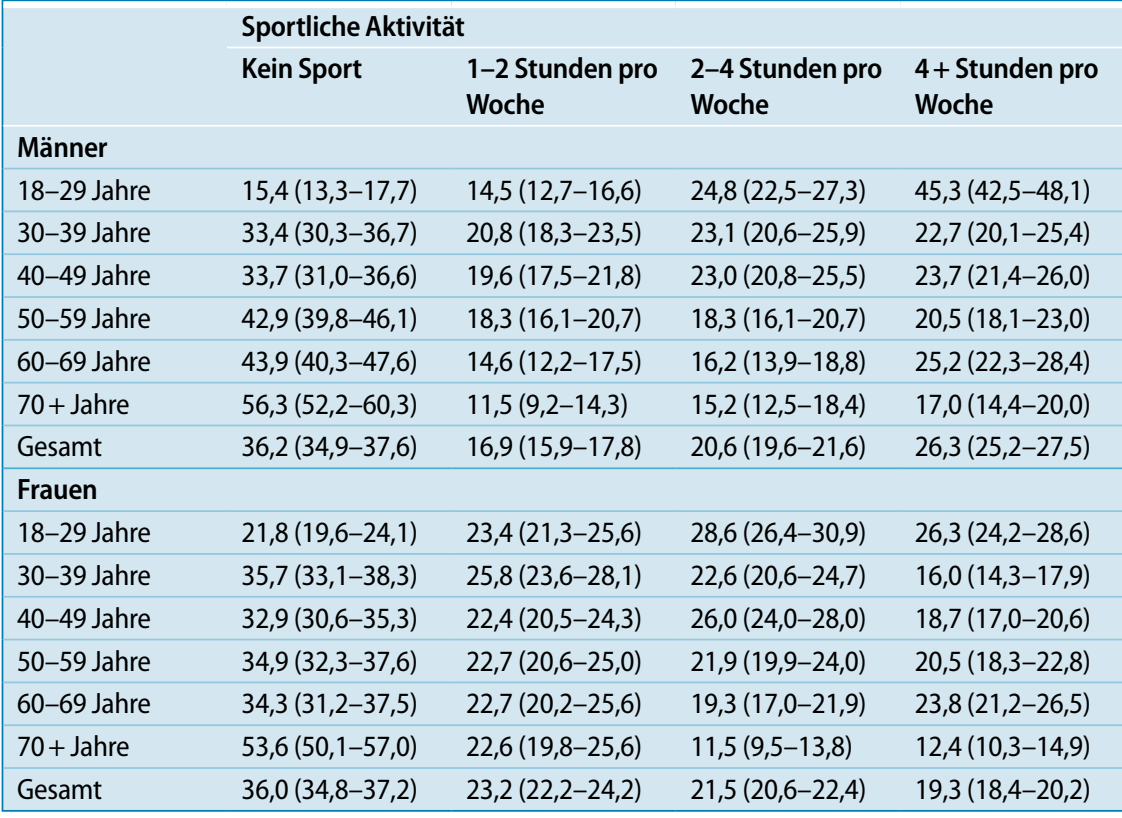

Tab. 4 Sportliche Aktivität in den letzten drei Monaten und Sozialstatus. Ergebnisse binär logistischer Regressionen bei Adjustierung bezüglich des Alters (Odds Ratios mit 95\%-Konfidenzintervallen und $p$-Werten) $(n=21.151)$

\section{Sozialstatus \\ Niedrig}

Mittel

OR (95\%-KI)

Männer

\begin{tabular}{llllll}
\hline 18-39 Jahre & Ref. & $1,76(1,27-2,44)$ & $\mathrm{p}<0,001$ & $4,66(3,16-6,87)$ & $\mathrm{p}<0,001$ \\
\hline 40-59 Jahre & Ref. & $1,89(1,41-2,53)$ & $\mathrm{p}<0,001$ & $4,20(3,09-5,72)$ & $\mathrm{p}<0,001$ \\
\hline 60+ Jahre & Ref. & $1,40(0,98-1,99)$ & $\mathrm{p}=0,051$ & $2,52(1,73-3,65)$ & $\mathrm{p}<0,001$ \\
\hline Gesamt & Ref. & $1,65(1,37-1,98)$ & $\mathrm{p}<0,001$ & $3,42(2,80-4,18)$ & $\mathrm{p}<0,001$ \\
\hline Frauen & & & & & \\
\hline 18-39 Jahre & Ref. & $2,03(1,57-2,64)$ & $\mathrm{p}<0,001$ & $3,88(2,85-5,28)$ & $\mathrm{p}<0,001$ \\
\hline 40-59 Jahre & Ref. & $2,27(1,78-2,90)$ & $\mathrm{p}<0,001$ & $5,14(3,95-6,70)$ & $\mathrm{p}<0,001$ \\
\hline 60+ Jahre & Ref. & $1,73(1,35-2,21)$ & $\mathrm{p}<0,001$ & $3,02(2,17-4,20)$ & $\mathrm{p}<0,001$ \\
\hline Gesamt & Ref. & $1,87(1,62-2,16)$ & $\mathrm{p}<0,001$ & $3,53(3,00-4,16)$ & $\mathrm{p}<0,001$ \\
\hline
\end{tabular}

zwischen den Angehörigen der niedrigen und mittleren Statusgruppe sind signifikante Unterschiede festzustellen, sodass von einem sozialen Gradienten bei der Sportausübung gesprochen werden kann. Dieser Gradient zeigt sich in allen betrachteten Altersgruppen. Lediglich bei den 60-jährigen und älteren Männern sind die Unterschiede zwischen der niedrigen und mittleren Statusgruppe nicht statistisch signifikant (• Tab. 4).

Im Bundes-Gesundheitssurvey 1998 und im telefonischen Gesundheitssurvey 2003 wurde die sportliche Aktivität in vergleichbarer Weise erhoben wie in der GEDA-Studie 2009. Dadurch kann die Entwicklung der Sportbeteiligung der erwachsenen Bevölkerung Deutschlands über die letzten zehn Jahre betrachtet werden. Allerdings muss die Analyse auf die Altersspanne von 18 bis 79 Jahre begrenzt werden, da die 8o-jährige und ältere Bevölkerung im Bundes-Gesundheitssurvey 1998 nicht berücksichtigt wurde. Neben Prävalenzen für Männer und Frauen und für verschiedene Altersgruppen sind in $\bullet$ Tab. 5 außerdem Odds Ratios mit den dazugehörigen 95\%-Konfidenzintervallen angegeben, die mittels binär logistischer Regressionen ermittelt wurden. Die Odds Ratios beziehen sich auf die Veränderung der Chance für sportli- 
Tab. 5 Entwicklung der sportlichen Aktivität im Zeitraum 1998 bis 2009. Prävalenzen in $\%$ mit 95\%-Konfindenzintervallen und altersadjustierte Odds Ratios (OR) mit 95\%-Konfidenzintervallen und $p$-Werten für die Veränderung der Chance für sportliche Aktivität im Jahr 2009 im Verhältnis zum Jahr 1998

\begin{tabular}{|llllll}
\hline & $\begin{array}{l}1998 \\
(\mathbf{n = 6 8 7 2 )}\end{array}$ & $\begin{array}{l}\mathbf{2 0 0 3} \\
(\mathbf{n = 8 1 5 8 )}\end{array}$ & $\begin{array}{l}\mathbf{2 0 0 9} \\
(\mathbf{n = 2 0 . 6 5 9 )}\end{array}$ & OR (95\%-KI) & p-Wert \\
\hline Männer & & & & & \\
\hline 18-29 Jahre & $72,9(68,9-76,6)$ & $73,3(68,8-77,3)$ & $84,6(82,3-86,7)$ & $2,05(1,60-2,64)$ & $\mathrm{p}<0,001$ \\
\hline 30-39 Jahre & $61,3(57,5-64,9)$ & $63,9(59,9-67,7)$ & $66,6(63,3-69,7)$ & $1,27(1,03-1,58)$ & $\mathrm{p}<0,050$ \\
\hline 40-49 Jahre & $58,4(54,2-62,4)$ & $60,9(57,1-64,6)$ & $66,3(63,4-69,0)$ & $1,42(1,16-1,74)$ & $\mathrm{p}<0,010$ \\
\hline 50-59 Jahre & $50,1(46,1-54,1)$ & $60,1(55,6-64,4)$ & $57,1(53,9-60,3)$ & $1,21(0,98-1,48)$ & $\mathrm{p}=0,072$ \\
\hline 60-69 Jahre & $45,4(40,6-50,4)$ & $53,5(48,8-58,1)$ & $56,1(52,4-59,7)$ & $1,49(1,17-1,89)$ & $\mathrm{p}<0,010$ \\
\hline 70-79 Jahre & $26,7(20,7-33,6)$ & $50,0(42,8-57,1)$ & $46,1(41,5-50,7)$ & $1,66(1,19-2,32)$ & $\mathrm{p}<0,010$ \\
\hline Gesamt & $56,0(54,2-57,9)$ & $61,4(59,6-63,3)$ & $64,6(63,2-65,9)$ & $1,45) 1,35-1,62)$ & $\mathrm{p}<0,001$ \\
\hline Frauen & & & & & \\
\hline 18-29 Jahre & $64,7(60,5-68,6)$ & $68,9(64,5-73,1)$ & $78,3(75,9-80,5)$ & $1,86(1,49-2,32)$ & $\mathrm{p}<0,001$ \\
\hline 30-39 Jahre & $57,0(53,2-60,7)$ & $59,6(55,6-63,4)$ & $64,4(61,7-66,9)$ & $1,36(1,13-1,64)$ & $\mathrm{p}<0,010$ \\
\hline 40-49 Jahre & $56,3(52,3-60,2)$ & $60,2(56,7-63,6)$ & $67,2(64,8-69,5)$ & $1,61(1,34-1,93)$ & $\mathrm{p}<0,001$ \\
\hline 50-59 Jahre & $53,5(49,4-57,6)$ & $62,7(58,5-66,8)$ & $65,2(62,5-67,8)$ & $1,53(1,26-1,86)$ & $\mathrm{p}<0,001$ \\
\hline 60-69 Jahre & $35,0(30,5-39,7)$ & $61,9(57,3-66,2)$ & $65,8(62,5-68,9)$ & $2,89(2,29-3,65)$ & $\mathrm{p}<0,001$ \\
\hline 70-79 Jahre & $24,9(19,9-30,7)$ & $46,9(41,0-52,9)$ & $49,8(45,7-53,9)$ & $2,27(1,70-3,05)$ & $\mathrm{p}<0,001$ \\
\hline Gesamt & $50,3(48,5-52,1)$ & $60,3(58,5-62,1)$ & $65,4(64,2-66,6)$ & $1,84(1,68-2,00)$ & $\mathrm{p}<0,001$ \\
\hline
\end{tabular}

che Aktivität im Jahr 2009 im Verhältnis zum Beginn des Beobachtungszeitraums im Jahr 1998 (Referenzkategorie). An den außerdem ausgewiesenen $\mathrm{p}$-Werten ist zu ersehen, ob sich die Chance für sportliche Aktivität im Beobachtungszeitraum statistisch signifikant verändert hat.

Für Männer ist in diesem Zeitraum ein Anstieg des Anteils an sportlich Aktiven von $56 \%$ auf $65 \%$ festzustellen, bei Frauen sogar von $50 \%$ auf $65 \%$. Eine altersdifferenzierte Betrachtung über die drei Beobachtungspunkte zeigt, dass im Zeitraum von 1998 bis 2003 insbesondere die Sportbeteiligung in den älteren Altersgruppen zugenommen hat, während im Zeitraum von 2003 bis 2009 der Anstieg in den jüngeren Altersgruppen am stärksten ausfiel. Die Ausnahmen stellen die 50- bis 59-jährigen und 70- bis 79-jährigen Männer dar, bei denen der Anteil an sportlich Aktiven in den letzten Jahren sogar wieder leicht rückläufig war. An den ausgewiesenen Odds Ratios und p-Werten ist zu erkennen, dass der Anstieg der sportlichen Aktivität in den meisten Altersgruppen statistisch signifikant war. Beispielsweise hat sich bei 18- bis 29-jährigen Männern und Frauen die Chance für sportliche Aktivität im Zeitraum von 1998 bis 2009 um den Faktor 2,05 beziehungsweise 1,86 erhöht (• Tab. 5).

\section{Diskussion}

Nach den Ergebnissen der GEDA-Studie 2009 sind $56 \%$ der 18 -jährigen und älteren Männer und 38\% der gleichaltrigen Frauen mindestens 2,5 Stunden pro Woche so aktiv, dass sie dabei ins Schwitzen oder außer Atem geraten. Die bis vor Kurzem geltenden Aktivitätsempfehlungen der CDC, denen zufolge Erwachsene an mindestens fünf Tagen in der Woche jeweils mindestens 30 Minuten körperlich aktiv sein sollten, werden von 23\% der Männer und $20 \%$ der Frauen erfüllt. Dass sie in den letzten drei Monaten Sport getrieben haben, trifft auf jeweils 64\% der Männer und Frauen zu. Ein Viertel der Männer und ein Fünftel der Frauen treiben wöchentlich sogar vier oder mehr Stunden Sport. Sowohl die körperliche als auch die sportliche Aktivität ist im jungen Erwachsenenalter am stärksten verbreitet und nimmt im Altersgang ab. Von den 70-Jährigen und Älteren sind noch ein Drittel der Männer und fast ein Viertel der Frauen mindestens 2,5 Stunden pro Woche körperlich aktiv. Sportlich betätigen sich in dieser Altersgruppe immerhin fast die Hälfte der Männer und Frauen.

Internationale Vergleiche werden dadurch erschwert, dass die körperliche und sportliche Aktivität in verschiedenen Stu- dien mit unterschiedlichen Instrumenten erhoben werden. Bezüglich der sportlichen Aktivität kann auf den Eurobarometer zurückgegriffen werden, einer von der EU-Kommission beauftragten Befragung, die in den EU-Mitgliedstaaten einheitlich durchgeführt wird [18]. Nach den Daten des Eurobarometers 2009 treiben in Deutschland 69\% der 15-jährigen und älteren Bevölkerung Sport. Dies stimmt recht gut mit den Ergebnissen der GEDA-Studie 2009 überein, wenngleich ein direkter Vergleich aufgrund der zusätzlichen Einbeziehung von Personen im Alter von 15 bis 17 Jahren, des deutlich kürzeren Erhebungszeitraums (Oktober 2009) und der abweichenden Formulierung der Fragen zur sportlichen Aktivität nicht möglich ist. Die Sportausübung von Erwachsenen liegt nach den Ergebnissen des Eurobarometers 2009 in Deutschland im europäischen Mittelfeld; die höchste Sportbeteiligung findet sich in den skandinavischen Ländern, die geringste in den südeuropäischen Ländern [18].

Die GEDA-Ergebnisse weisen außerdem auf Unterschiede in der körperlichen und sportlichen Aktivität nach dem sozialen Status hin. Bei der körperlichen Aktivität sprechen diese dafür, dass Männer und Frauen mit niedrigem Sozialstatus häufiger aktiv sind als diejenigen mit hohem Sozialstatus. Dies gilt zumindest für das junge und mittlere Erwachsenenalter (vergleiche [19]). Ein Grund hierfür dürfte sein, dass Personen mit niedrigem Sozialstatus häufiger in körperlich beanspruchenden Berufen tätig sind, während Personen mit hohem Sozialstatus zu einem größeren Anteil sitzenden Tätigkeiten nachgehen. Dafür spricht auch eine weiterführende Analyse, in der der relative Einfluss der statusbildenden Indikatoren Bildung, berufliche Stellung und Einkommen untersucht wurde. Demnach haben Männer und Frauen mit einer niedrigen beruflichen Stellung eine um etwa den Faktor zwei erhöhte Chance, mindestens 2,5 Stunden in der Woche körperlich aktiv zu sein, während sich der Einfluss der Bildung und des Einkommens bei gleichzeitiger Berücksichtigung der beruflichen Stellung als statistisch nicht bedeutsam erwies. Auch der dargestellte Befund, dass bei den 6o-Jährigen und Älteren die körperliche Akti- 
vität in der höheren Statusgruppe stärker verbreitet ist als in der niedrigen Statusgruppe, unterstützt diese Annahme.

Noch deutlicher fallen die statusspezifischen Unterschiede in der sportlichen Aktivität aus. Männer und Frauen mit hohem Sozialstatus treiben mehr als dreimal häufiger Sport als diejenigen mit niedrigem Sozialstatus. Die Angehörigen der mittleren Statusgruppe sind ebenfalls zu einem größeren Anteil sportlich aktiv als die Angehörigen der niedrigen Statusgruppe, allerdings nicht im gleichen Maße wie die Angehörigen der hohen Statusgruppe. In Bezug auf die Sportbeteiligung kann somit von einem sozialen Gradienten gesprochen werden: Je höher der soziale Status, desto häufiger wird Sport getrieben. Dieser Gradient ist bei Männern und Frauen in allen betrachteten Altersgruppen zu beobachten (vergleiche [20]).

Unter Einbeziehung von Daten des Bundes-Gesundheitssurveys 1998 und des telefonischen Gesundheitssurveys 2003 konnte des Weiteren gezeigt werden, dass die Sportbeteiligung in den letzten zehn Jahren zugenommen hat. Im Zeitraum von 1998 bis 2003 ist insbesondere der Anstieg in den höheren Altersgruppen auffällig, was unter anderem auf die Zunahme von spezifischen Sportangeboten für diese Altersgruppe zurückgeführt werden kann [21, 22]. Für den Zeitraum von 2003 bis 2009 ist vor allem für die jüngeren Altersgruppen ein erheblicher Anstieg der sportlichen Aktivität festzustellen. Aussagen über zeitliche Entwicklungen und Trends bei der körperlichen Aktivität sind mit den Daten des RKI zurzeit noch nicht möglich, da diese in den früheren Gesundheitssurveys nicht beziehungsweise nicht in vergleichbarer Weise erhoben wurden [23]. Außerdem haben sich die Aktivitätsempfehlungen immer wieder geändert. In der GEDAStudie 2009 wurde von den Empfehlungen der CDC aus dem Jahr 1996 ausgegangen. Mittlerweile wurden diese überarbeitet unter Berücksichtigung unterschiedlicher Voraussetzungen und Bedarfe im Kindes- und Jugendalter ( 5 bis 17 Jahre), jungen und mittleren Erwachsenenalter (18 bis 64 Jahre) und höherem Lebensalter (65+ Jahre) [2, 24]. Vor diesem Hintergrund stellt die kontinuierliche Beobachtung der körperlichen Akti- vität eine große Herausforderung für das Gesundheitsmonitoring am RKI dar.

Bei der Bewertung und Einordnung der vorgestellten Ergebnisse ist zu berücksichtigen, dass diese auf Selbstangaben zur körperlichen und sportlichen Aktivität beruhen, die Ungenauigkeiten aufweisen und Fehlerquellen unterliegen können. Hierunter fallen unter anderem Erinnerungslücken, die Möglichkeit der Überschätzung der eigenen Aktivitäten, insbesondere da es sich um ein sozial erwünschtes Verhalten handelt, sowie Unterschiede in der Definition der Begriffe körperliche Aktivität und Sport. Auch die allgemein gehaltene Formulierung der Fragen zur körperlichen und sportlichen Aktivität kann zu einem unterschiedlichen Antwortverhalten führen. Aufgrund des zur Verfügung stehenden Zeitfensters von etwa einer halben Stunde für das komplette Interview und des breiten Themenspektrums der Befragung war es im Rahmen der GEDA-Studie nicht möglich, den Befragten am Telefon eine Definition von Sport zu vermitteln oder Sportarten einzeln zu erfassen. Damit wurde es letztlich den Studienteilnehmern überlassen, was sie unter Sport verstehen, wobei diesbezügliche Unterschiede nach Alter, Geschlecht und auch sozialem Status denkbar und sogar wahrscheinlich sind. Ebenso kann es bezüglich der körperlichen Aktivität zu Variationen im Antwortverhalten kommen, auch aufgrund von Unterschieden in der körperlichen Leistungsfähigkeit und Fitness.

Trotz dieser Einschränkungen weisen die Ergebnisse der GEDA-Studie zahlreiche Ansatzpunkte für die Bewegungsund Sportförderung auf. $\mathrm{Zu}$ verweisen ist insbesondere auf die Bevölkerungsgruppen, die sich bislang nur wenig oder gar nicht körperlich und sportlich betätigen. Immerhin 19\% der 18-jährigen und älteren Männer und 26\% der gleichaltrigen Frauen sind an keinem Tag in der Woche körperlich so aktiv, dass sie dabei ins Schwitzen oder außer Atem geraten. Das Potenzial für die Bewegungsförderung ist aber noch deutlich größer, da die Aktivitätsempfehlungen der CDC von mehr als drei Viertel der erwachsenen Bevölkerung nicht erreicht werden. Eine ähnliche Schlussfolgerung lässt sich in Bezug auf die sportliche Aktivität ziehen, da mehr als ein Drittel der Männer und Frauen überhaupt keinen Sport macht. Neben alters- und geschlechtsspezifischen Unterschieden sollten auch die statusspezifischen Unterschiede berücksichtigt werden, um bewegungs- und sportfördernde Maßnahmen und Programme zielgruppenorientiert planen und umsetzen zu können.

\section{Korrespondenzadresse \\ Dr. T. Lampert}

FG27 Gesundheitsberichterstattung,

Robert Koch-Institut

General-Pape-Str. 62-66, 12101 Berlin

t.lampert@rki.de

Interessenkonflikt. Der korrespondierende Autor gibt an, dass kein Interessenkonflikt besteht.

\section{Literatur}

1. Department of Health (2004) Physical activity health improvement and prevention: at least five a week. Department of Health, London

2. US Department of Health and Human Services (USDHHS) (2008) 2008 physical activity guidelines for americans. USDHHS, Washington DC. http:// www.health.gov/paguidelines (letzter Zugriff 30.6.2011)

3. Warburton DER, Charlesworth S, Ivey A et al (2010) A systematic review of the evidence for Canada's physical activity guidelines for Adults. Int J Behav Nutr Phys Act 7:39

4. Samitz G, Egger M, Zwahlen M (2011) Domains of physical activity and all-cause mortality: systematic review and dose-response meta-analysis of cohort studies. Int J Epidemiol:1-19 (advanced access)

5. Teychenne M, Ball K, Salmon J (2008) Physical activity and likelihood of depression in adults: a review. Prev Med 46(5):397-411

6. Robert Koch-Institut (Hrsg) (2005) Körperliche Aktivität. Gesundheitsberichterstattung des Bundes Heft 26. Robert Koch-Institut, Berlin

7. Rommel A, Klaes L, Cosler D et al (2008) Lebensführung und Sport. Beiträge zur Gesundheitsberichterstattung des Bundes. Robert Koch-Institut, Berlin

8. Kurth BM, Lange C, Kamtsiuris P et al (2009) Gesundheitsmonitoring am Robert Koch-Institut. Bundesgesundheitsbl Gesundheitsforsch Gesundheitsschutz 52(5):557-570

9. Robert Koch-Institut (Hrsg) (2011) Daten und Fakten: Ergebnisse der Studie "Gesundheit in Deutschland aktuell 2009". Beiträge zur Gesundheitsberichterstattung des Bundes. Robert Koch-Institut, Berlin

10. Gabler S, Häder S (1999) Generierung von Telefonstichproben mit TelSuSa. ZUMA-Nachrichten 44:138-143

11. ADM/BVM (1998) Richtlinie für telefonische Befragungen. Telefonstichproben in Deutschland. In: Gabler S, Häder S, Hoffmeyer-Zlotnik JHP (Hrsg) Telefonstichproben in Deutschland. Westdeutscher Verlag, Opladen, S 181-187 
12. Heckel C (2001) Erstellung der ADM-Telefonauswahlgrundlage. ZUMA-Workshop Methodische Probleme bei der Stichprobenziehung und Realisierung. Aschpurwitz + Behrens GmbH, Mannheim

13. The American Association for Public Opinion Resarch (AAPOR) (2008) Standard definition: final dispositions of case codes and outcome rates for surveys. http://www.aapor.org/AM/Template. $\mathrm{cfm} ?$ Section $=$ Standard_Definitions\&Template $=$ / $\mathrm{CM} /$ ContentDisplay.cfm\&ContentID $=1273$ (letzter Zugriff 30.06.2011)

14. Müters S, Lippe E von der, Kamtsiuris P et al (2010) Dokumentation zur Response in der Studie Gesundheit in Deutschland aktuell 2009. http://www. rki.de/cln_178/nn_201180/DE/Content/GBE/Erhebungen/Gesundheitsurveys/Geda/response geda09, templateld $=$ raw, property $=$ publicationFile.pdf/responsegeda09.pdf (letzter Zugriff 30.06.2011)

15. Pate RR, Pratt M, Blair SN et al (1995) Physical activity and public health: a recommendation from the centers for disease control and prevention and the American College of Sports Medicine. JAMA 272:402-407

16. Lampert T, Kroll LE (2009) Die Messung des sozioökonomischen Status in sozialepidemiologischen Studien. In: Richter M, Hurrelmann K (Hrsg) Gesundheitliche Ungleichheit. Grundlagen, Probleme, Perspektiven, 2. aktualisierte Aufl. VS Verlag für Sozialwissenschaften, Wiesbaden, S 309-334

17. UNESCO (2003) International standard classification of education. In: Hoffmeyer-Zlotnik JHP, Wolf C (Hrsg) Advances in cross-national comparision. Kluwer, New York, S 195-220

18. European Commission (2010) Sport and physical activity. Special Eurobarometer 334/Wave 72.3. TNS Opinion \& Social. http://ec.europa.eu/public opinion/archives/ebs/ebs_334_en.pdf (letzter Zugriff 30.06.2011)

19. Mensink GBM (2002) Körperliches Aktivitätsverhalten in Deutschland. In: Samitz G, Mensink GBM (Hrsg) Körperliche Aktivität in Prävention und Therapie. Hans Marseille, München, S 35-44

20. Lampert $T$ (2010) Tabakkonsum, sportliche Inaktivität und Adipositas. Assoziationen mit dem sozialen Status. Dtsch Arztebl 107(1-2):1-7

21. Mechling H (2003) Gesundheitssport im Alter. Public Health Forum 11(41):22-23

22. Lampert T, Mensink GBM, Ziese T (2005) Sport und Gesundheit bei Erwachsenen in Deutschland. Bundesgesundheitsbl Gesundheitsforsch Gesundheitsschutz 48:1357-1364

23. Mensink GBM (1999) Körperliche Aktivität. Gesundheitswesen 61(Sonderheft 2):S126-S131

24. Weltgsundheitsorganisation (WHO) (2010) Global recommendations on physical activity for health. http://whqlibdoc.who.int/publications/2010/9789241599979_eng.pdf (letzter Zugriff: 30.06.2011)

\section{GIB AIDS KEINE CHANCE aktuell}

Neue Ausgabe des Newsletters der BZgA zum Welt-Aids-Tag 2011 erschienen

Stigmatisierung und Diskriminierung von Menschen mit HIV und Aids abzubauen, ist das zentrale Anliegen der diesjährigen Welt-Aids-Tags-Kampagne „Positiv zusammen leben. Aber sicher!". Sie wird gemeinsam vom Bundesministerium für Gesundheit, der Bundeszentrale für gesundheitliche Aufklärung (BZgA), der Deutschen AIDS-Hilfe und der Deutschen AIDS-Stiftung durchgeführt. Noch immer erfahren viele HIV-positiv-Getestete massive Nachteile bei einer Offenlegung ihrer HIV-Infektion. Wie die aktuelle Studie der BZgA zum Thema „Schwule Männer und Aids" zeigt, wurde jeder fünfte HIV-positive schwule Mann schon mehrfach sozial gemieden. Viele befürchten zudem wegen der eigenen HIV-Infektion schlechter als andere behandelt zu werden. $81 \%$ der HIVpositiv-Getesteten haben es aus Angst vor Diskriminierung schon ein- oder mehrmals vermieden, ihre Infektion offen zu thematisieren. Angesichts dieser Ergebnisse, über die in der neuen Ausgabe des Newsletters "GIB AIDS KEINE CHANCE aktuell" berichtet wird, ist die Solidaritäts- und Antidiskriminierungskampagne zum Welt-Aids-Tag wichtiger denn je. Denn ein offener und solidarischer Umgang mit HIV-Infizierten und an Aids erkrankten Menschen ist eine wesentliche Grundlage für erfolgreiche Prävention.

In einem weiteren Themenschwerpunkt geht es um sexuell übertragbare Infektionen (STI). Da sie eine HIV-Übertragung begünstigen können, erweitert die BZgA ihre HIV-/Aidsprävention um Informationen über Infektionsrisiken und Schutzmöglichkeiten von STI. Zwar zeichnet sich nach der aktuellen BZgA-Repräsentativerhebung „Aids im öffentlichen Bewusstsein" bei den Befragten eine gestiegene Sensibilisierung für die Risiken von STI ab, aber noch immer besteht diesbezüglich ein hoher Informationsbedarf. Deshalb ist es umso wichtiger, die verschiedenen Zielgruppen mit den Informationen der Aidsprävention und der Prävention von STI zu erreichen.
Der Newsletter "GIB AIDS KEINE CHANCE aktuell ${ }^{\prime \prime}$ informiert in regelmäßigen $A b$ ständen über die Arbeit der BZgA und über aktuelle Entwicklungen in der Aidsprävention. Er steht zum Download unter www. bzga.de/presse/hintergrundinformationen und kann kostenlos per E-mail unter order@bzga.de, per Fax unter 0221-8992257 sowie unter www.bzga.de/infomaterialien/ aidsaufklaerung/ bestellt werden.

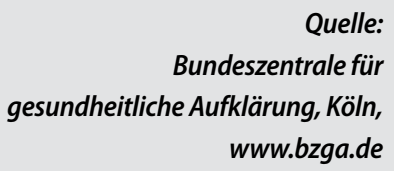

Quelle: www.bzga.de 